\title{
Prevalência de disfunção temporomandibular em pacientes com hemofilia
}

\author{
Thatiana B. Guimarães ', Mariana B. F. Cabrini', Sonia M. B. Guimarães ${ }^{7}$, Antônio S. Guimarães ${ }^{1}$, \\ Camila Q. Leite', Sandra V. Antunes², Luis G. Alonso ${ }^{3}$ \\ 'Cirurgião dentista- Ambulatório de Disfunção Temporomandibular e Dor Orofacial/Unifesp, \\ ${ }^{2}$ Hematologista - Ambulatório de Hemofilia/Unifesp, ${ }^{3}$ Médico- Professor Adjunto da Disciplina de Anatomia \\ Descritiva e Topográfica do Departamento de Morfologia e Genética/Unifesp
}

Guimarães $T^{1}$, Cabrini $M B^{1}$, Guimarães $S M^{1}$, Guimarães $A S^{1}$, Leite $C^{1}{ }^{1}$, Antunes SV² Alonso $L^{3}$. Prevalência de disfunção temporomandibular em pacientes com hemofilia. 2012;3(4):225-6

\section{INTRODUÇÃO E OBJETIVOS}

Coagulopatias hereditárias são doenças hemorrágicas resultantes da deficiência quantitativa e/ou qualitativa de uma ou mais proteínas plasmáticas (fatores) da coagulação. Entre as coagulopatias hereditárias, as mais comuns são a hemofilia e a doença de Von Willebrand. A hemofilia é um distúrbio hereditário da coagulação resultante de herança genética ligada ao cromossomo $X$ recessiva e, como tal, é transmitida quase exclusivamente a indivíduos do gênero masculino por meio de mãe portadora (maioria dos casos) aparentemente normal ou ainda por mutações novas. Clinicamente, caracteriza-se por sangramentos, os quais podem ocorrer em qualquer estrutura ou órgão do indivíduo acometido. Pode ser devida à deficiência do fator VIII, condição esta denominada hemofilia $A$ ou, deficiência do fator IX conhecida como hemofilia B. Clinicamente, ambas as entidades são indistinguíveis.

A hemofilia A ocorre em cerca de 1:10.000 indivíduos do gênero masculino. As manifestações hemorrágicas podem ocorrer já no primeiro ano de vida e sua gravidade depende dos níveis plasmáticos do fator VIII.
As hemorragias podem se apresentar de diversas formas: espontâneas ou precedidas por traumas. As hemorragias podem se expressar clinicamente como hematúria, epistaxe, hematêmese, melena ou por meio de hematomas, sangramentos retroperitoniais e intraarticulares (hemartroses), que constituem um dos aspectos mais característicos das formas graves da doença.

A classificação da hemofilia A varia de acordo com o nível de atividade coagulante do fator VIII (fator VIII:C), sendo o nível de normalidade definido como $1 \mathrm{UI} / \mathrm{ml}$ de fator VIII:C (100\%). Recomenda-se classificar os pacientes como: graves, aqueles que possuem fator VIII:C inferior a $1 \%$ do normal ou $<0,01 \mathrm{UI} / \mathrm{ml}$; moderados, aqueles que possuem fator VIII: C entre 1\%$5 \%$ do normal ou 0,01 - $0.05 \mathrm{UI} / \mathrm{ml} \mathrm{e}$; leves, aqueles que possuem fator VIII:C $>5 \%-<40 \%$ do normal ou $>0,05-<0,40 \mathrm{UI} / \mathrm{ml}$.

As características da hemofilia $B$ são semelhantes quanto ao quadro clínico e classificação em relação à forma $A$, da qual difere quanto ao fator plasmático deficiente que, neste caso, é o fator IX. A hemofilia B apresenta uma incidência 3-4 vezes menor que a hemofilia A. 
À exceção do hematoma superficial, a hemorragia intra-articular é a complicação mais comum da hemofilia. As articulações mais afetadas em ordem decrescente são: joelhos, cotovelos, tornozelos, pés, quadris e ombros. A articulação temporomandibular (ATM) parece ser raramente envolvida.

Episódios de hemartrose, nas articulações das extremidades em pacientes com hemofilia, são bem documentados. Entretanto, hemartrose na ATM (e no compartimento estomatognático) em pacientes com coagulopatias parecem ser eventos raros ou, talvez, não tão bem documentados.

Uma das complicações mais prevalentes da hemofilia é a artropatia crônica, que resulta de repetidos sangramentos na articulação, podendo levar à limitação de movimento. A limitação de movimento mandibular pode ser um dos sinais dos pacientes que apresentam disfunção temporomandibular (DTM) que, por definição, é um termo coletivo que abrange um largo espectro de problemas clínicos da articulação e dos músculos da área orofacial, sendo tal disfunção caracterizada por dor, ruídos nas ATM e função mandibular irregular ou limitada. A DTM é considerada um subgrupo distinto das desordens musculoesqueléticas e reumatológicas, e representa uma causa importante de dor não dental na região orofacial.

Os sinais e sintomas da DTM são extremamente comuns. Estudos epidemiológicos sugerem que 40\% a $75 \%$ da população geral possui algum sinal de DTM. A população adulta jovem, de 20 a 40 anos, é a mais atingida. A prevalência de DTM é maior entre indivíduos de 20 a 50 anos. $O$ objetivo deste estudo foi verificar a prevalência de disfunção temporomandibular em pacientes com hemofilias $A$ e $B$.

\section{MÉTODOS}

Foram avaliados 39 pacientes hemofílicos do gênero masculino (18-51 anos) com idade mediana de 26 anos e 79 controles do gênero masculino (1851 anos) com idade mediana de 27 anos. Os indivíduos foram submetidos ao questionário da Academia Europeia das Desordens Craniomandibulares para avaliação da presença de sintomas de DTM. Aqueles que responderam afirmativamente a, pelo menos, uma pergunta foram submetidos aos eixos I e II do RDC (critério diagnóstico para pesquisa em disfunção temporomandibular). A análise estatística foi realizada com o teste do qui-quadrado considerando-se um nível de significância $p \leq 0,05$.

\section{RESULTADOS}

Sinais ou sintomas de hemartrose na ATM não foram verificados na casuística estudada e não foram observadas diferenças significantes entre os grupos avaliados.

\section{CONCLUSÕES}

A articulação temporomandibular é muito solicitada com cargas elevadas durante a mastigação e apertamentos. No entanto, a hemartrose da ATM em pacientes com coagulonopatias parece ser rara. A presença de DTM tem sido pouco avaliada em pacientes com hemofilia. Os sinais e sintomas associados à ATM não apresentaram significância estatística na comparação do grupo controle em relação ao grupo de estudo.

\section{REFERÊNCIAS}

1. Alpkiliç Baskirt E, Ak G, Zulfikar B. Oral and general healthrelated quality of life among young patients with haemophilia. Haemophilia. 2009; 15(1):193-8.

2. Antunes SV. Hemorragia intracraniana em hemofílicos A: Análise de 36 episódios. [Tese Mestrado]. São Paulo. Escola Paulista de Medicina, 1992.

3. Dworkin SF, LeResche L. Research diagnostic criteria for temporomandibular disorders: review, criteria, examinations and specifications, critique. J Craniomandib Disord. 1992 Fall; 6(4):301-55.

4. Greer RB, Ballard JO. Musculoskeletal bleeding in hemophilia. Pediatr Ann. 1982;1 1:521-7.

5. Hitchings EJ. The oral health of individuals with haemophilia: a review of the literature. N Z Dent J. 2011;107(1):4-11.

6. Soucie JM, Cianfrini C, Janco RL, Kulkarni R, Hambleton J, Evatt B, et al. Joint range-of-motion limitations among young males with hemophilia: prevalence and risk factors. Blood. 2004;103(7):2467-73.

7. Nishioka GJ, Van Sickels JE, Tilson HB. Hemophilic arthropathy of the temporomandibular joint: review of the literature, a case report, and discussion. Oral Surg Oral Med Oral Pathol. 1988; 65(2): 145-150. 